Urologe $2021 \cdot 60: 149-150$

https://doi.org/10.1007/s00120-021-01446-3

Angenommen: 8. Januar 2021

(c) Springer Medizin Verlag GmbH, ein Teil von Springer Nature 2021

\author{
A. Haferkamp ${ }^{1} \cdot$ Christian G. Stief $^{2}$ \\ ${ }^{1}$ Klinik und Poliklinik für Urologie und Kinderurologie, Universitätsmedizin der Johannes Gutenberg- \\ Universität Mainz, Mainz, Deutschland \\ ${ }^{2}$ Klinik und Poliklinik für Urologie, Ludwig Maximilians-Universität München, Campus Großhadern, \\ München, Deutschland
}

\title{
Aspekte des perioperativen Managements
}

Der Urologe beschäftigt sich in diesem Monatsheft mit dem Thema Qualität bei unterschiedlichen urologischen Eingriffen, insbesondere mit Qualitätskriterien, der Messung von Outcome-Qualität und den daraus resultierenden Konsequenzen. Der Themenbereich chirurgische Qualität an sich ist für die Urologen nicht neu und begleitet uns bereits seit Jahren, nimmt aber eine immer größere Bedeutung ein. Neben der hohen Relevanz für unsere Patienten ist die Erfassung und Auswertung von Qualitätsparametern auch für die Ausbildung und Weiterentwicklung eines Operateurs von zentraler Bedeutung und hat zudem ökonomische Aspekte, die in Teilen bereits im DRG-System abgebildet sind. Zudem verfolgt die Gesundheitspolitik immer häufiger den Ansatz eines „value-based healthcare“, so dass dieser Themenkomplex nicht nur für unsere Patienten eine hohe Relevanz hat, sondern auch aus ökonomischer Sicht zukünftig immer wichtiger wird.

Anhand verschiedener urologischer Erkrankungen zeigen die Autoren dieses Heftes auf, wo wir derzeit mit unseren Qualitätskriterien, der Qualitätserfassung und Auswertung stehen und welche Konsequenzen wir daraus ziehen sollten.

Roghmann et al. identifizieren anhand eines der größten urologischen Eingriffe, der radikalen Zystektomie, die ja bekanntlich mit einer relevanten Morbidität und Mortalität einhergeht, chirurgische Qualitätsparameter, die eine Relevanz für onkologisch/funktionelle Ergebnisse und Komplikationsfreiheit haben. Dazu gehören beispielsweise das Erreichen eines negativen Absetzungsrandes, die korrekte Durchführung einer Lymphadenektomie, die Anlage einer kontinenten Harnableitung oder der Erhalt der Sexualfunktion.

Schulz et al. und Fischer et al. erweitern die Qualitätsbetrachtung bei der radikalen Zystektomie noch um den gesamten perioperativen Bereich und geben einerseits einen Literaturüberblick über die derzeitige Evidenz zu „enhanced recovery after surgery“ (ERAS) bei der radikalen Zystektomie und zeigen ein Beispiel für die interdisziplinäre Umsetzung eines solchen standardisierten Konzepts an der Universitätsmedizin Mainz.

Grabbert et al. weisen in ihrer Arbeit am Beispiel der Harninkontinenz auf unterschiedliche Einschlusskriterien und uneinheitliche Definitionen für Therapieerfolge der vorliegenden Studien hin, die eine Vergleichbarkeit und qualitative Bewertung erschweren bis verhindern. Gerade im Bereich der männlichen Belastungsinkontinenz fehlen zudem vergleichende Studien der vorhandenen chirurgischen Verfahren, so dass wir uns seit Jahren lediglich auf dem Niveau von Expertenmeinungen bewegen. An dieser Stelle setzt das neu zwischen den Fachgesellschaften etablierte Zertifizierungssystem der Kontinenz- und Beckenbodenzentren an, das die Qualität der Inkontinenzbehandlung verbessern will und deshalb erstmals die Abfrage von Ergebnisqualität von Standardeingriffen in seine Zertifizierungsbedingungen aufgenommen hat.

Eine ähnlich schwierige Situation findet sich bei der seltenen Tumorentität Peniskarzinom. Thomas et al. zeigen eine große Schwankungsbreite in der Versor- 
gungsqualität und hieraus resultierenden häufig suboptimalen onkologischen und funktionellen Behandlungsergebnissen auf. Eine Zentrumsbildung könnte hier ein Schlüssel zur Verbesserung der Behandlungsqualität sein - wie in manchen Regionen gezeigt.

Würnschimmel et al. zeigen am Beispiel des lokalisierten Prostatakarzinoms auf, wie eine medizinische Qualitätssicherung und -weiterentwicklung erfolgen sollte und welche Parameter von Bedeutung sind. Schlüsselkriterien sind standardisierte „patient reported outcome measurements" (PROM) zur Ergebnisdokumentation und die Verlagerung von komplexen Krankheitsbildern in dezidierte Zentren mit High-volumeChirurgie.

Neben den krankenhausinternen Maßnahmen zur Qualitätssicherung und -verbesserung können Kliniken aber auch die Möglichkeiten einer externen Qualitätssicherung nutzen wie beispielsweise unabhängige Zertifizierungen durch professionelle Zertifizierungseinrichtungen. Hierzu zählt auch die von Tritschler vorgestellte „Initiative Qualitätsmedizin“, die eine Qualitätsmessung auf der Basis von Routinedaten durchführt, eine Transparenz durch Veröffentlichung dieser Daten herstellt und mögliche Verbesserungen der Qualität durch einen nachfolgenden Peer ReviewProzess erarbeitet.

Dieses Themenheft verdeutlicht einmal mehr, wie unterschiedlich unser Fachgebiet bei den verschiedenen urologischen Erkrankungen in Bezug auf Qualitätskriterien, ihre Erfassung und Auswertung aufgestellt ist, zeigt gleichzeitig aber Tools auf, wie dies deutlich verbessert werden kann.

Wir wünschen viel Spaß beim Lesen!

Axel Haferkamp, Christian Stief

\section{Korrespondenzadresse

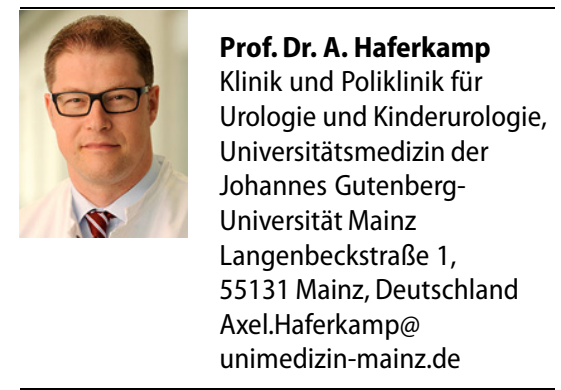

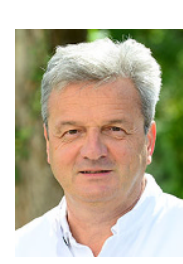

Prof. Dr. Christian G. Stief, MD, PhD

Klinik und Poliklinik

für Urologie, Ludwig

Maximilians-Universität

München, Campus

Großhadern

Marchioninistr. 15, 81377 München, Deutschland christian.stief@ med.uni-muenchen.de

Interessenkonflikt. A. Haferkamp und C. G. Stief geben an, dass kein Interessenkonflikt besteht.
Hier steht eine Anzeige. Springer 